\title{
Funcionalidad del injerto renal a 1 año del trasplante renal
}

\author{
Kidney graft functionality 1 year after kidney transplantation
}

\begin{abstract}
Juan C. H. Hernández-Rivera ${ }^{1 *}$, Ramón Espinoza-Pérez², José Cruz-Santiago ${ }^{3}$, Raúl Rodríguez-Gómez², Guillermo Meza-Jiménez³, Jorge D. Cancino-López², Martha Cruz-López², M. Juana Pérez-López³, Marlene Santos-Caballero ${ }^{4}$, Mariana Salazar-Mendoza ${ }^{5}$ y Ramón Paniagua-Sierra ${ }^{1}$

'Unidad de Investigación Médica en Enfermedades Nefrológicas, Hospital de Especialidades Centro Médico Nacional Siglo XXI, Instituto Mexicano del Seguro Social (IMSS); ${ }^{2}$ Unidad de Trasplante Renal, Hospital de Especialidades Centro Médico Nacional Siglo XXI, IMSS; ${ }^{3}$ Unidad de Trasplante Renal, Hospital de Especialidades Centro Médico Nacional La Raza, IMSS; '4nidad de Trasplante Renal, Hospital General Centro Médico Nacional La Raza, IMSS; ${ }^{5}$ Hospital Regional Lic. Adolfo López Mateos, Instituto de Seguridad y Servicios Sociales de los Trabajadores del Estado. Ciudad de México, México
\end{abstract}

\section{Resumen}

Objetivo: Determinar la funcionalidad del injerto renal a 1 año en una cohorte retrospectiva en México. Método: Cohorte de trasplante renal de 2013 a 2017 en México. Se analizaron 1118 pacientes. Se estudiaron cinco desenlaces: supervivencia global, supervivencia del injerto renal, supervivencia del paciente, función retardada y disfunción aguda. Para la supervivencia del injerto renal se usó Kaplan-Meier. Para el riesgo se realizó análisis bivariado y multivariado con valor significativo $p<0.05$. Resultados: De los 1118 pacientes con trasplante renal, 57 (5.09\%) tuvieron pérdida del injerto, 52 (4.65 \%) fallecieron durante el año de seguimiento; la supervivencia del paciente fue del $95.35 \%$ y la supervivencia del injerto fue del $90.25 \%$. Conclusiones: Los factores de riesgo para los desenlaces fueron trasplante de donante fallecido, receptor mayor de 50 años y uso de agentes policlonales. Las infecciones y la edad están relacionadas con la muerte del paciente.

Palabras clave: Trasplante renal. Supervivencia. Mortalidad. Función retardada.

\begin{abstract}
Objective: To determine the evolution, associated factors and the outcomes of transplanted people one year after the evolution of a cohort in Mexico. Method: Kidney transplant cohort from 2013 to 2017 in Mexico. 1118 patients were analyzed. Five outcomes were studied: overall survival, kidney graft, patient survival, delayed function, and acute dysfunction. Kaplan-Meier was used for kidney graft survival. For risk, bivariate and multivariate analyzes were performed with a significant value of $p<0.05$. Results: Of the 1118 kidney transplant patients, 57 (5.09\%) had kidney graft loss, 52 (4.65\%) died during the oneyear follow-up; survival of the patient of $95.35 \%$ and of the graft $90.25 \%$. Conclusions: The risk factors for the outcomes were transplantation from a deceased donor, recipient over 50 years of age and use of polyclonal agents. Infections and age are related to the death of the patient.
\end{abstract}

Keywords: Kidney transplant. Survival. Mortality. Delayed function.

\footnotetext{
Correspondencia:

*Juan C. H. Hernández-Rivera

Avda. Cuauhtémoc, 330

Col. Doctores

Fecha de recepción: 17-09-2020

C.P. 06722, Ciudad de México, México

E-mail: juancarloshhernandezrivera@ hotmail.com

Cir Cir. 2022;90(1):90-99

Contents available at PubMed www.cirugiaycirujanos.com 0009-7411/○ 2021 Academia Mexicana de Cirug
} 


\section{Introducción}

El trasplante renal es considerado como la mejor modalidad terapéutica para los pacientes con enfermedad renal crónica terminal'1. Las ventajas del trasplante renal son la reincorporación a las actividades laborales y a la vida familiar, la mejor calidad de vida y la mejor relación costo-beneficio en comparación con cualquier modalidad de diálisis ${ }^{2-5}$.

El trasplante renal no está exento de desventajas, y entre ellas, la disponibilidad de donantes es una de las más importantes ${ }^{5}$. En el caso de tener potenciales donadores vivos relacionados, la selección requiere, además de la compatibilidad inmunológica, las evaluaciones necesarias que garanticen la salud del donante 6 . En México, después de la creación del Centro Nacional de Trasplantes (CENATRA), se ha observado una necesidad creciente de órganos y tejidos, de acuerdo con los registros ${ }^{7}$. El proceso puede prolongarse cuando la población tiene características epidemiológicas poco favorables, como son prevalencias elevadas de obesidad, diabetes o hipertensión. En la población mexicana, estas enfermedades tienen unas tasas de prevalencia de las más elevadas del mun$\mathrm{do}^{8-11}$. Un estudio de Amato et al..$^{12}$ mostró que la población en México con enfermedad renal crónica terminal tenía una prevalencia de 1142 por millón de habitantes en el año 2005, y además se ha establecido que de forma general la enfermedad renal crónica tiene una prevalencia del $14.7 \%$ en todos los estadios $^{13}$.

Cuando el paciente requiere donante cadavérico, la permanencia en lista de espera puede prolongarse cuando, por factores culturales, religiosos o legales, la tasa de donación es baja ${ }^{14}$. En México, la disponibilidad de donantes cadavéricos es baja por todas las razones anteriores. La espera prolongada incrementa la mortalidad de los pacientes y puede superar a la mortalidad de los que se encuentran en diálisis crónica sin expectativa de trasplante renal.

El monitoreo de los programas de trasplante renal es útil en la detección de oportunidades para incrementar el número de procedimientos y mejorar las tasas de resultados exitosos ${ }^{15-18}$. El monitoreo es particularmente importante en poblaciones como la mexicana, que tiene una incidencia elevada de enfermedad renal crónica terminal, muchas veces sin un diagnóstico, por lo cual se ha desarrollado enfermedad renal crónica ${ }^{19-21}$, además de poca disponibilidad de donantes vivos y cadavéricos?.
El objetivo de este estudio es conocer la tasa de desenlaces adversos, como mortalidad, pérdida del injerto, función renal retardada, rechazo, disfunción aguda del injerto y supervivencia, así como los factores relacionados con ellos.

\section{Método}

\section{Diseño}

Se estudió una cohorte retrospectiva de pacientes que recibieron trasplante renal en dos hospitales pertenecientes a la red del Instituto Mexicano del Seguro Social en la Ciudad de México: el Centro Médico Nacional Siglo XXI y el Hospital de Especialidades del Centro Médico Nacional La Raza.

\section{Pacientes}

Se consideraron elegibles los pacientes que recibieron un trasplante renal en el periodo entre el 1 de enero de 2013 y el 31 de diciembre de 2017. Se incluyeron aquellos que tenían los datos clínicos y bioquímicos necesarios para el análisis en la etapa basal y durante el seguimiento por 12 meses. Se excluyeron los pacientes con información incompleta o que perdieron la cobertura de la seguridad social.

\section{Obtención de datos}

Se obtuvieron los datos somatométricos, demográficos, clínicos y de comorbilidad de cada uno de los pacientes, a partir de sus expedientes, por personal específicamente capacitado. Se recabó toda la información de variables relacionadas con el trasplante, como tipo de trasplante renal, esquema de inducción (el cual está en relación con el riesgo inmunológico pretrasplante, siendo de dos tipos: bajo riesgo con basiliximab, que es un agente monoclonal, y alto riego con timoglobulina, un agente policlonal) e inmunosupresión primaria, tiempos de isquemia fría y caliente, y creatinina al egreso. Se registraron las variables bioquímicas en los meses 1, 3, 6, 9 y 12 postrasplante, así como los eventos adversos, las hospitalizaciones y los días de hospitalización.

\section{Estadística}

Los datos se presentan como promedios y desviación estándar (DE) para las variables continuas, o 
como frecuencias o medianas y rangos intercuartíli$\cos (R I Q)$ para las variables nominales o de libre distribución. Para los análisis de supervivencia su utilizaron curvas de Kaplan-Meier y el análisis de factores de riesgo se realizó con regresión logística, bivariado y multivariado. La significancia estadística se estableció en $p<0.05$. Para el análisis se empleó el paquete estadístico SPSSw v25.

\section{Resultados}

Se incluyeron 1118 pacientes de las dos unidades de trasplante renal. Los datos demográficos, clínicos y bioquímicos más relevantes se muestran en la tabla 1. Debe señalarse que se trató de una cohorte de pacientes jóvenes, la mayoría hombres, y el donante vivo fue el más frecuente. La creatinina basal fue de $1.14 \mathrm{mg} / \mathrm{dl}$ (RIQ: 0.92-1.43). Hubo un número importante de pacientes con anemia (hemoglobina $11.9 \mathrm{~g} / \mathrm{dl}$; RIQ: 10.6-13.2). Se usaron agentes policlonales en 404 pacientes ( 315 de donante fallecido y 89 de donante vivo, con panel reactivo alto $o$ anticuerpos donante-específicos presentes).

En los 12 meses de seguimiento, de los 1118 pacientes hubo 52 muertes, 57 tuvieron pérdidas del injerto, 64 tuvieron función retardada del injerto, 103 tuvieron disfunción aguda del injerto no relacionada con rechazo y 122 tuvieron rechazo, ya fuera de tipo humoral, celular o mixto. Al transcurso del año de seguimiento en la cohorte de 1118 pacientes, 1066 (95.35\%) sobrevivían y la funcionalidad del injerto renal era buena en 1009, descartando los pacientes que fallecieron y aquellos que tuvieron pérdida del injerto renal y pasaron a otra modalidad de terapia de remplazo renal, como diálisis peritoneal o hemodiálisis (90.25\%) (Figs. 1 and 2).

De la totalidad de la población, la mediana de edad fue de 29 años (RIQ: 24-40), 803 trasplantes (71.8\%) fueron de donante vivo y $315(28.2 \%)$ de donante fallecido, y la distribución por sexo fue de 659 hombres (58.9\%) y 459 mujeres (41.1\%) (Fig. 2).

En relación con los cinco desenlaces, los resultados fueron:

- La función retardada del injerto renal se presentó en 64 casos, de los cuales 13 (20.31\%) fueron de donante vivo y $51(79.69 \%)$ de donante fallecido, asociado sobre todo a los tiempos prolongados de isquemia fría y a donantes con criterios expandidos.

- La disfunción aguda del injerto, no asociada a rechazo, se presentó en 103 pacientes en algún
Tabla 1. Características basales de los 1118 pacientes con trasplante renal

\begin{tabular}{|c|c|c|c|}
\hline Variable & Mediana & RIQ 25-75 & Media (DE) \\
\hline Edad (años) & 29 & $24-40$ & $33.25(12.11)$ \\
\hline Peso (kg) & 63.3 & $54-72$ & $63.76(12.82)$ \\
\hline Talla (m) & 1.63 & $1.56-1.69$ & $1.62(0.09)$ \\
\hline $\mathrm{Cr}(\mathrm{mg} / \mathrm{dL}) 1$. $^{\mathrm{er}} \mathrm{mes}$ & 1.14 & $0.92-1.43$ & $1.42(1.43)$ \\
\hline $\begin{array}{l}\mathrm{AU}(\mathrm{mg} / \mathrm{dL}) 1 .{ }^{\mathrm{er}} \\
\text { mes }\end{array}$ & 4.5 & $3.7-5.5$ & $4.73(1.53)$ \\
\hline $\mathrm{Hb}(\mathrm{g} / \mathrm{dL}) 1$ er mes $^{\text {er }}$ & 11.9 & $10.6-13.2$ & $11.87(2.02)$ \\
\hline $\mathrm{Ca}(\mathrm{mg} / \mathrm{dL}) 1 .^{\mathrm{er}} \mathrm{mes}$ & 9.4 & $9.0-9.9$ & $9.38(0.88)$ \\
\hline $\mathrm{P}(\mathrm{mg} / \mathrm{dL}) 1$. $^{\mathrm{er}} \mathrm{mes}$ & 2.6 & $1.9-3.5$ & $2.8(1.24)$ \\
\hline PTH 1. er mes & 99.2 & $31.8-265.4$ & 216.9 (303.09) \\
\hline Variable & & Número & Porcentaje \\
\hline \multicolumn{4}{|c|}{ Tipo de trasplante renal } \\
\hline $\begin{array}{l}\text { Donante vivo } \\
\text { Donante fallecido }\end{array}$ & & $\begin{array}{l}803 \\
315\end{array}$ & $\begin{array}{l}71.8 \\
28.2\end{array}$ \\
\hline \multicolumn{4}{|l|}{ Sexo } \\
\hline Masculino & & 659 & 41.1 \\
\hline Femenino & & 459 & 58.9 \\
\hline \multicolumn{4}{|l|}{ Dicotómico por edad } \\
\hline Menos de 50 años & & 979 & 87.6 \\
\hline Más de 50 años & & 139 & 12.4 \\
\hline
\end{tabular}

momento de la evolución en los 12 meses; cuatro de estos pacientes ya habían cursado con función retardada del injerto. De los pacientes con disfunción aguda del injerto, 10 tuvieron pérdida del injerto, uno de ellos falleció y 92 están con injerto funcional. Algunas características observadas en este grupo de pacientes fueron más de donante fallecido, hombres e índice de masa corporal $>25 \mathrm{~kg} / \mathrm{m}^{2}$.

- El rechazo se presentó en 122 pacientes, dos de ellos inclusive con dos eventos de rechazo. De estos 122 pacientes con rechazo, 13 ya habían tenido función retardada del injerto, 34 tuvieron pérdida del injerto renal y 3 muerte asociada a dicho evento de rechazo por ser de tipo humoral. Finalmente, 85 pacientes de los 122 se mantuvieron a los 12 meses con injerto funcional. Pacientes con uso de inducción policlonal por riesgo inmunológico alto.

- La pérdida del injerto renal se observó en 57 pacientes en el tiempo de vigilancia de 12 meses, y se vio relacionado con condiciones propias del injerto, en 34 pacientes con rechazo, en 


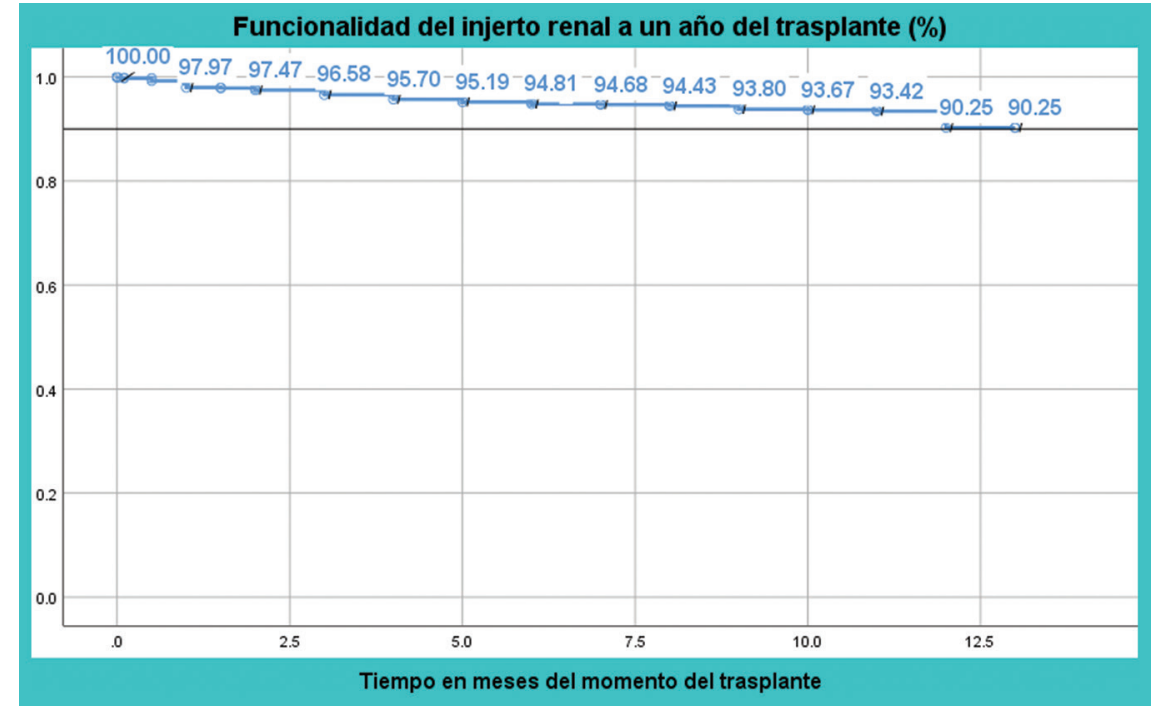

Figura 1. Funcionalidad (supervivencia) del injerto renal al año de evolución.

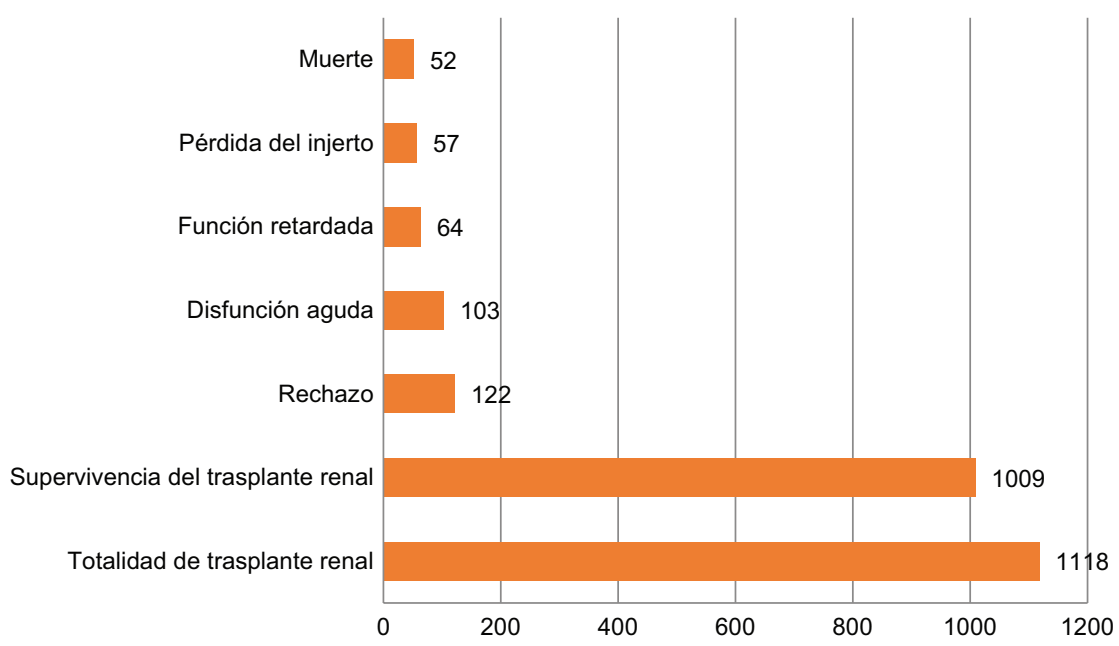

Figura 2. Número de pacientes por desenlaces clínicos importantes al año de evolución.

13 con función retardada del injerto y en 10 con disfunción aguda del injerto. Solo uno falleció. Cinco de estos pacientes tuvieron una serie de eventos múltiples, iniciando con función retardada del injerto y posteriormente sin un orden establecido, pero pasando por disfunción aguda, rechazo y finalmente pérdida del injerto. El caso de muerte fue un rechazo humoral no relacionado con el antígeno leucocitario humano, con pérdida del injerto, y un par de días después con neumonía y muerte.

- La muerte ocurrió en 52 pacientes, 3 de ellos que anteriormente habían tenido función retardada del injerto, 3 con rechazo de tipo humoral y 1 con disfunción aguda del injerto. Sin embargo, lo que estuvo más relacionado con la muerte fueron los procesos infecciosos, en el siguiente orden: 17 pacientes tuvieron neumonía atípica, 6 neumonía bacteriana, 4 choque séptico relacionado con neumonía, 3 sepsis abdominal, 3 sepsis, 1 neumonía por Pseudomonas, 1 neumonía con coriocarcinoma de pulmón, 1 infarto agudo al miocardio, 1 neumonía con infección urinaria grave, 1 pancreatitis, 1 choque séptico con influenza, 1 absceso abdominal; 1 paciente falleció en casa a las 2 semanas del trasplante sin tener determinada la 
causa de defunción, y 8 más de causa no clara con respuesta inflamatoria sistémica sin proceso infeccioso identificado, sin rechazo.

En las tablas 2 y 3 se hace una separación de los 1009 pacientes con injerto funcional a los 12 meses en grupos separados por tres condiciones: sexo (masculino y femenino), tipo de donante (vivo o fallecido) y edad (<50 años y > 50 años). La distribución de los injertos funcionales fue: 590 hombres (recibieron trasplante 659 , correspondiente al $89.52 \%$ de los hombres con injerto funcional) y 419 mujeres (459 injertos iniciales para este rubro, $91.28 \%$ para este sexo). En cuanto al tipo de donante, a los 12 meses se mantenían con injerto funcional 755 de donante vivo (se operaron 803 , el $94.02 \%$ de la totalidad de donante vivo funcional al año) y 254 de donante fallecido (315 fueron los receptores de trasplante de donante fallecido, $80.63 \%$ ). Por último, en relación a la edad, estaban funcionales 882 menores de 50 años (979 operados, $90.09 \%$ ) y 127 mayores de 50 años al momento del trasplante (139 fueron los receptores de trasplante, $91.36 \%$ ) (Tabla 2).

La distribución por sexo, tipo de trasplante y edad para cada uno de los desenlaces se muestra en la tabla 3.

En relación con la edad, de los pacientes que tenía injerto funcional, en comparación con aquellos que fallecieron o regresaron a terapia de reemplazo renal (pérdida del injerto renal), se observó que los pacientes que fallecieron tuvieron una media de edad de 44.24 años (DE: 14.11), frente a una media de los pacientes con pérdida del injerto de 33.63 años (DE: 13.68), contrastando con los pacientes con injerto funcional a los 12 meses, de 33.25 años (DE: 12.11) (Fig. 3).

Se estudiaron cinco desenlaces principales (Tabla 4): mortalidad, pérdida del injerto renal, función retardada del injerto, disfunción aguda del injerto renal y supervivencia. Estos desenlaces se combinaron con nueve factores: donante fallecido, edad > 50 años del receptor al momento del trasplante renal, sexo femenino, índice de masa corporal $>25 \mathrm{~kg} / \mathrm{m}^{2}$, fracción de eyección del ventrículo izquierdo $(\mathrm{FEVI})<50 \%$, disfunción diastólica de algún grado previa al trasplante renal e inducción con agente policlonal (por indicación del riesgo inmunológico). Se realizó análisis bivariado en la primera parte de cada tabla y multivariado en el apartado inferior de cada tabla correspondiente.

Los resultados fueron los siguientes:
Tabla 2. Características al mes del trasplante renal, dicotomizado

\begin{tabular}{|c|c|c|c|}
\hline Variable & $\begin{array}{c}\text { Hombres (659) } \\
\text { Mediana (RIQ 25-75) }\end{array}$ & $\begin{array}{c}\text { Mujeres (459) } \\
\text { Mediana (RIQ 25-75) }\end{array}$ & $p\left({ }^{*}\right)$ \\
\hline Edad (años) & $30(24-41)$ & $31(25-41)$ & 0.474 \\
\hline Peso (kg) & 68 (60.5-75.5) & $55(49-63)$ & 0.001 \\
\hline Talla (m) & $1.67(1.62-1.71)$ & $1.55(1.50-1.60)$ & 0.001 \\
\hline $\begin{array}{l}\mathrm{Cr}(\mathrm{mg} / \mathrm{dL}) 1 \text { er }^{\text {mes }} \\
\text { me }\end{array}$ & $1.30(1.10-1.57)$ & $0.97(0.80-1.16)$ & 0.001 \\
\hline $\begin{array}{l}\mathrm{AU}(\mathrm{mg} / \mathrm{dL}) 1{ }^{\mathrm{er}} \\
\mathrm{mes}\end{array}$ & $4.8(4.0-5.7)$ & $4.2(3.4-5.1)$ & 0.001 \\
\hline $\mathrm{Hb}(\mathrm{g} / \mathrm{dL}) 1$. $^{\mathrm{er}} \mathrm{mes}$ & $12.2(10.7-13.6)$ & $11.4(10.3-12.6)$ & 0.483 \\
\hline $\begin{array}{l}\mathrm{Ca}(\mathrm{mg} / \mathrm{dL}) 1 \text { er }^{\text {er }} \\
\text { mes }\end{array}$ & $9.5(9.0-10.0)$ & $9.4(9.0-9.9)$ & 0.429 \\
\hline $\mathrm{P}(\mathrm{mg} / \mathrm{dL})$ 1. er mes & $2.5(1.8-3.5)$ & $2.8(2.1-3.5)$ & 0.153 \\
\hline $\begin{array}{l}\text { PTH }(p g / m L) ~ 1 e r ~ \\
\text { mes }\end{array}$ & 92 (61.35-238.32) & $138(25.9-277.4)$ & 0.441 \\
\hline
\end{tabular}

\section{Donante vivo (803) Donante fallecido $\mathrm{p}^{*}$}

(315)

\begin{tabular}{|c|c|c|c|}
\hline Edad (años) & $28(23.25-36.00)$ & 37 (29-48) & 0.001 \\
\hline Peso (kg) & $62(53-71)$ & $65(55-74)$ & 0.619 \\
\hline Talla (m) & $1.64(1.56-1.70)$ & $1.62(1.55-1.68)$ & 0.249 \\
\hline $\begin{array}{l}\mathrm{Cr}(\mathrm{mg} / \mathrm{dL}) 1{ }^{\mathrm{er}} \\
\text { mes }\end{array}$ & $1.1(0.90-1.33)$ & $1.30(1.05-1.78)$ & 0.001 \\
\hline $\begin{array}{l}\mathrm{AU}(\mathrm{mg} / \mathrm{dL}) 1{ }^{\text {er }} \\
\text { mes }\end{array}$ & $4.4(3.7-5.2)$ & $5.0(4.0-6.3)$ & 0.005 \\
\hline $\mathrm{Hb}(\mathrm{g} / \mathrm{dL})$ 1. er mes & $12.1(10.0-13.5)$ & $10.9(9.2-12.4)$ & 0.001 \\
\hline $\begin{array}{l}\mathrm{Ca}(\mathrm{mg} / \mathrm{dL}) 1 .^{\text {er }} \\
\text { mes }\end{array}$ & $9.5(9-10)$ & $9.2(8.5-9.8)$ & 0.001 \\
\hline $\mathrm{P}(\mathrm{mg} / \mathrm{dL}) 1^{\text {er }}$ mes & $2.8(2.0-3.5)$ & $2.4(1.7-3.2)$ & 0.023 \\
\hline \multirow{2}{*}{$\begin{array}{l}\text { PTH }(p g / m L) 1{ }^{e r} \\
\text { mes }\end{array}$} & $85.7(31.25-203.20)$ & $124(66.49-522.30)$ & 0.441 \\
\hline & $<50$ años (979) & $\geq 50$ años (139) & $\mathrm{p}^{*}$ \\
\hline Peso (kg) & $62(53-71.5)$ & $67(59.75-75.00)$ & 0.137 \\
\hline Talla (m) & $1.63(1.55-1.69)$ & $1.62(1.56-1.68)$ & 0.353 \\
\hline $\begin{array}{l}\mathrm{Cr}(\mathrm{mg} / \mathrm{dL}) 1 .^{\mathrm{er}} \\
\text { mes }\end{array}$ & $1.13(0.92-1.40)$ & $1.14(0.90-1.51)$ & 0.378 \\
\hline $\begin{array}{l}\mathrm{AU}(\mathrm{mg} / \mathrm{dL}) 1 \text {. }^{\text {er }} \\
\text { mes }\end{array}$ & $4.59(3.8-5.5)$ & $4.3(2.56-5.6)$ & 0.011 \\
\hline $\mathrm{Hb}(\mathrm{g} / \mathrm{dL})$ 1. er mes & $11.9(10.6-13.3)$ & $11.3(9.9-13.0)$ & 0.087 \\
\hline $\begin{array}{l}\mathrm{Ca}(\mathrm{mg} / \mathrm{dL}) 1 \text { er }^{\text {er }} \\
\text { mes }\end{array}$ & $9.5(9-10)$ & $9.3(8.6-9.7)$ & 0.013 \\
\hline $\mathrm{P}(\mathrm{mg} / \mathrm{dL})$ 1. ${ }^{\text {er }} \mathrm{mes}$ & $2.7(1.9-3.5)$ & $2.5(1.75-3.50)$ & 0.316 \\
\hline $\begin{array}{l}\text { PTH }(p g / m L) 1 .{ }^{\text {er }} \\
\text { mes }\end{array}$ & $\begin{array}{c}103.10 \\
(30.75-274.40)\end{array}$ & 115 (85.5-419.9) & 0.439 \\
\hline
\end{tabular}

AU: ácido úrico; Ca: calcio; Cr: creatinina; DE: desviación estándar; Hb: hemoglobina; P: fósforo; PTH: paratohormona; RIQ: rango intercuartílico.

${ }^{*}$ Prueba $\chi^{2}$. 
Tabla 3. Factores de riesgo relacionados con los desenlaces en trasplante renal

\begin{tabular}{|c|c|c|c|c|c|}
\hline Variable & Total (1118) & Muerte (52) & $p^{*}$ & Pérdida del injerto (57) & $\mathbf{p}^{*}$ \\
\hline $\begin{array}{l}\text { Sexo } \\
\text { Hombres } \\
\text { Mujeres }\end{array}$ & $\begin{array}{l}659(58.95) \\
459(41.05)\end{array}$ & $\begin{array}{l}32(61.53) \\
20(38.47)\end{array}$ & 0.083 & $\begin{array}{l}37(64.91) \\
20(35.09)\end{array}$ & 0.233 \\
\hline $\begin{array}{l}\text { Tipo de donante } \\
\text { Vivo } \\
\text { Fallecido }\end{array}$ & $\begin{array}{l}803(71.82) \\
315(28.18)\end{array}$ & $\begin{array}{l}17(32.69) \\
35(67.31)\end{array}$ & 0.001 & $\begin{array}{l}26(45.61) \\
31(54.39)\end{array}$ & 0.567 \\
\hline $\begin{array}{l}\text { Edad } \\
\qquad 50 \text { años } \\
\geq 50 \text { años }\end{array}$ & $\begin{array}{l}979(87.56) \\
139(12.44)\end{array}$ & $\begin{array}{l}44(84.61) \\
8(15.39)\end{array}$ & 0.508 & $\begin{array}{c}53(92.98) \\
4(7.02)\end{array}$ & 0.218 \\
\hline Variable & & Función retardada (64) & $\mathbf{p}^{*}$ & Injerto funcional (1009) & $\mathbf{p}^{*}$ \\
\hline $\begin{array}{l}\text { Sexo } \\
\text { Hombres } \\
\text { Mujeres }\end{array}$ & $\begin{array}{l}803(71.82) \\
315(28.18)\end{array}$ & $\begin{array}{l}44(68.75) \\
20(31.25)\end{array}$ & 0.573 & $\begin{array}{l}755(74.82) \\
254(25.18)\end{array}$ & 0.001 \\
\hline $\begin{array}{l}\text { Tipo de donante } \\
\text { Vivo } \\
\text { Fallecido }\end{array}$ & $\begin{array}{l}659(58.94) \\
459(41.06)\end{array}$ & $\begin{array}{l}13(20.31) \\
51(79.69)\end{array}$ & 0.001 & $\begin{array}{l}590(58.47) \\
419(41.53)\end{array}$ & 0.330 \\
\hline $\begin{array}{l}\text { Edad } \\
\quad<50 \text { años } \\
\geq 50 \text { años }\end{array}$ & $\begin{array}{l}979(87.56) \\
139(12.44)\end{array}$ & $\begin{array}{c}61(95.31) \\
3(4.69)\end{array}$ & 0.053 & $\begin{array}{l}882(87.41) \\
127(12.59)\end{array}$ & 0.635 \\
\hline
\end{tabular}

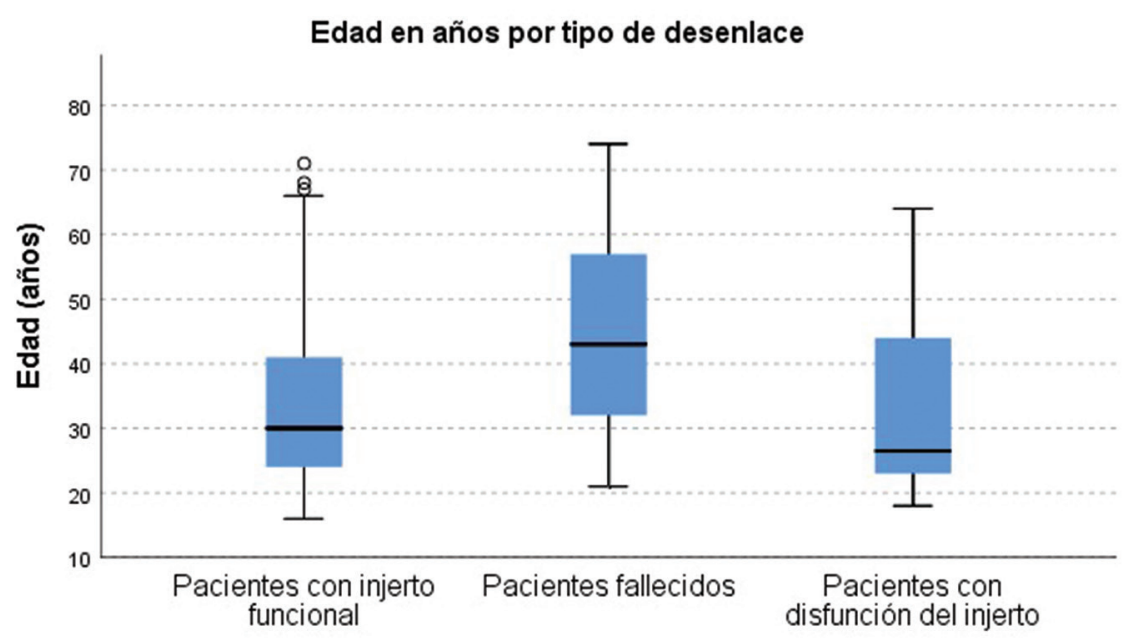

Figura 3. Diferencia de edades entre los grupos de pacientes con injerto funcional, fallecidos y con pérdida del injerto. Con diferencia por prueba de Kruskal-Wallis entre los grupos $(p=0.001)$.

- Para la mortalidad, los factores de riesgo estadísticamente significativos asociados en el análisis bivariado fueron donante fallecido y edad $>50$ años, mismos que continuaron siendo significativos en el análisis multivariado.

- Para el desenlace de pérdida del injerto, ninguno de los factores estuvo relacionado con riesgo o protección.
- La función retardada tuvo significancia para riesgo en el análisis bivariado en caso de donante fallecido, edad > 50 años, índice de masa corporal > $25 \mathrm{~kg} / \mathrm{m}^{2} \mathrm{e}$ inducción con agente policlonal.

- La disfunción aguda del injerto en el transcurso de los 12 meses de seguimiento mostró dos factores significativos en el análisis bivariado: un 
Tabla 4. Análisis de factores para cada uno de los desenlaces en trasplante renal a 1 año

\begin{tabular}{|c|c|c|c|c|c|c|}
\hline \multirow[t]{2}{*}{ Variable/desenlace } & OR & IC95\% & $\mathrm{p}$ & OR & IC95\% & $p$ \\
\hline & \multicolumn{3}{|c|}{ Análisis bivariado } & \multicolumn{3}{|c|}{ Análisis multivariado } \\
\hline \multicolumn{7}{|l|}{ Mortalidad } \\
\hline Donante fallecido & 4.26 & $2.10-8.63$ & 0.001 & 2.34 & $1.00-5.47$ & 0.050 \\
\hline Edad > 50 años & 4.16 & $2.07-8.37$ & 0.001 & 4.01 & $1.85-8.71$ & 0.001 \\
\hline Femenino & 0.94 & $0.45-1.78$ & 0.904 & 1.06 & $0.52-2.15$ & 0.859 \\
\hline$I M C>25$ & 1.17 & $0.60-2.27$ & 0.645 & 1.26 & $0.61-2.57$ & 0.525 \\
\hline $\mathrm{Hb}<12 \mathrm{~g} / \mathrm{dl}$ & 0.79 & $0.40-1.53$ & 0.490 & 0.89 & $0.43-1.83$ & 0.760 \\
\hline Uso previo de EPO & 0.81 & $0.41-1.63$ & 0.568 & 0.64 & $0.29-1.39$ & 0.263 \\
\hline $\mathrm{FEVI}<50 \%$ & 1.09 & $0.37-3.17$ & 0.866 & 1.12 & $0.33-3.74$ & 0.851 \\
\hline Disfunción diastólica & 1.18 & $0.59-2.33$ & 0.631 & 1.21 & $0.56-2.62$ & 0.616 \\
\hline Inducción policlonal & 3.18 & $1.54-6.53$ & 0.002 & 2.05 & $0.86-4.88$ & 0.104 \\
\hline \multicolumn{7}{|c|}{ Función retardada del injerto } \\
\hline Donante fallecido & 8.62 & $4.08-18.21$ & 0.001 & 13.85 & $5.21-36.76$ & 0.001 \\
\hline Edad $>50$ años & 2.40 & $1.19-4.80$ & 0.013 & 1.08 & $0.47-2.44$ & 0.851 \\
\hline Femenino & 0.65 & $0.34-1.25$ & 0.207 & 0.69 & $0.35-1.36$ & 0.289 \\
\hline $\mathrm{IMC}>25$ & 2.40 & $1.30-4.45$ & 0.005 & 1.90 & $0.98-3.67$ & 0.055 \\
\hline $\mathrm{Hb}<12 \mathrm{~g} / \mathrm{dl}$ & 0.66 & $0.36-1.21$ & 0.180 & 0.70 & $0.36-1.36$ & 0.294 \\
\hline Uso previo de EPO & 0.67 & $0.35-1.28$ & 0.232 & 0.66 & $0.32-1.35$ & 0.259 \\
\hline $\mathrm{FEVI}<50 \%$ & 1.41 & $0.58-3.45$ & 0.445 & 2.26 & $0.77-6.61$ & 0.135 \\
\hline Disfunción diastólica & 1.06 & $0.56-2.00$ & 0.841 & 1.28 & $0.61-2.70$ & 0.507 \\
\hline Inducción policlonal & 1.99 & $1.02-3.68$ & 0.028 & 2.36 & $1.02-5.46$ & 0.044 \\
\hline \multicolumn{7}{|c|}{ Disfunción aguda del injerto } \\
\hline Donante fallecido & 1.55 & $0.95-2.52$ & 0.078 & 1.18 & $0.63-2.24$ & 0.593 \\
\hline Edad > 50 años & 0.63 & $0.28-1.41$ & 0.265 & 0.64 & $0.27-1.49$ & 0.643 \\
\hline Femenino & 0.57 & $0.33-0.97$ & 0.040 & 0.49 & $0.28-0.84$ & 0.010 \\
\hline $\mathrm{IMC}>25$ & 0.82 & $0.50-1.36$ & 0.463 & 0.74 & $0.44-1.25$ & 0.268 \\
\hline $\mathrm{Hb}<12 \mathrm{~g} / \mathrm{dl}$ & 0.91 & $0.56-1.47$ & 0.703 & 0.74 & $0.44-1.24$ & 0.260 \\
\hline Uso previo de EPO & 0.43 & $0.24-0.75$ & 0.003 & 0.37 & $0.20-0.68$ & 0.001 \\
\hline $\mathrm{FEVI}<50 \%$ & 0.79 & $0.33-1.88$ & 0.595 & 0.61 & $0.23-1.59$ & 0.315 \\
\hline Disfunción diastólica & 1.00 & $0.60-1.66$ & 0.990 & 1.02 & $0.58-1.79$ & 0.920 \\
\hline Inducción policlonal & 1.72 & $1.05-2.79$ & 0.028 & 1.57 & $0.84-2.91$ & 0.151 \\
\hline \multicolumn{7}{|c|}{ Disfunción aguda del injerto } \\
\hline Donante fallecido & 2.67 & $1.66-4.30$ & 0.001 & 2.00 & $1.09-3.67$ & 0.025 \\
\hline Edad > 50 años & 3.06 & $1.79-5.24$ & 0.001 & 3.17 & $1.75-5.73$ & 0.001 \\
\hline Femenino & 0.83 & $0.51-1.36$ & 0.479 & 0.84 & $0.50-1.39$ & 0.511 \\
\hline $\mathrm{IMC}>25$ & 0.97 & $0.59-1.57$ & 0.901 & 0.73 & $0.43-1.22$ & 0.232 \\
\hline $\mathrm{Hb}<12 \mathrm{~g} / \mathrm{dl}$ & 1.21 & $0.75-1.95$ & 0.430 & 1.26 & $0.75-2.13$ & 0.373 \\
\hline Uso previo de EPO & 0.66 & $0.41-1.10$ & 0.112 & 0.63 & $0.36-1.10$ & 0.111 \\
\hline $\mathrm{FEVI}<50 \%$ & 1.33 & $0.68-2.83$ & 0.360 & 1.27 & $0.56-2.87$ & 0.552 \\
\hline Disfunción diastólica & 1.33 & $0.82-2.16$ & 0.237 & 1.26 & $0.73-2.17$ & 0.401 \\
\hline Inducción policlonal & 1.91 & $1.18-3.08$ & 0.008 & 1.36 & $0.74-2.48$ & 0.309 \\
\hline
\end{tabular}

EPO: eritropoyetina; FEVI: fracción de eyección del ventrículo izquierdo; Hb: hemoglobina; IC95\%: intervalo de confianza al 95\%; IMC: índice de masa corporal; OR: odds ratio.

factor protector, que fue el sexo femenino y se mantuvo en el análisis multivariado, y un factor de riesgo, solo en el análisis bivariado, que fue la inducción con un agente policlonal.

- Por último, la supervivencia mostró como factores de riesgo el donante fallecido, el receptor mayor de 50 años al momento del trasplante y la inducción con agente policlonal, continuando con la significancia estadística solo los dos primeros factores.

\section{Discusión}

Los datos obtenidos en nuestro estudio son muy variados debido a que fueron cinco los desenlaces estudiados (retardo en la función del injerto, disfunción aguda, rechazo, supervivencia del injerto renal y supervivencia del paciente) y siete los factores potenciales influyentes; se excluyeron los factores ya comprobados, como la edad del donante y la proteinuria postrasplante, entre otros ${ }^{22}$. La inducción con un 
agente policlonal fue el factor de mayor riesgo para los desenlaces de mortalidad (odds ratio [OR]: 3.18; intervalo de confianza al 95\% [IC95\%]: 1.54-6.53), seguido de la función retardada (OR: 1.99; IC95\%: 1.02-3.68), la disfunción aguda (OR: 1.72; IC95\%: 1.05-2.79) y la supervivencia del trasplante (OR: 1.91; IC95\%: 1.183.08). Hay que ser cautelosos con la interpretación de estos resultados, ya que son pacientes con alto riesgo inmunológico (sensibilizados, altamente sensibilizados, fallecidos, etc.); sin embargo, nos obliga a pensar que también serán pacientes más susceptibles a presentar infecciones (complicación comprobada que influye en la función del injerto, enfermedades sistémicas y pérdida asociada, sobre todo por citomegalovirus, virus herpes zóster, virus Epstein-Barr, poliomavirus, etc. $)^{23,24}$ y rechazo, que requieren una profilaxis postrasplante inmediata, con una vigilancia inmunológica y clínica más estrecha debido a los resultados obtenidos en este estudio, similares a los de otros estudios realizados en México ${ }^{25}$.

La mortalidad estuvo mayormente relacionada con infecciones asociadas con sepsis, y la supervivencia del injerto renal mantuvo un nexo con la función retardada del injerto, la disfunción aguda y el rechazo. Este dato contrasta un poco con los de otros estudios que relacionan la supervivencia del injerto y la mortalidad con la función retardada; en nuestro estudio, en cambio, la función retardada solo se relacionó con la supervivencia del injerto renal ${ }^{26}$. La disfunción del injerto estuvo más relacionada en el donante fallecido durante el primer año y existió una fuerte correlación con una mejor calidad del riñón trasplantado basado en el índice de perfil de donantes de riñón (KDPI, Kidney donor profile index ${ }^{27}$. Existen registros muy contundentes en otros países; por ejemplo, en los Estados Unidos de América cuentan con una base de datos donde tienen registrada la supervivencia y, por ende, la mortalidad ${ }^{28}$. En el caso de la supervivencia para el donante vivo tienen cifras del $98.8 \%$ a los 12 meses del trasplante, contrastando con nuestra cohorte, en la que la supervivencia con este tipo de donante es del $97.43 \%$ (fallecieron 17 de 659 receptores de donante vivo a los 12 meses). Del mismo modo, la supervivencia en caso de donante fallecido a los 12 meses es del $93.8 \%$, frente al $92.38 \%$ en nuestros pacientes ( 35 defunciones de 459 pacientes receptores de trasplante de donante fallecido) ${ }^{28}$.

El trasplante de donante fallecido es otro de los factores con mayor impacto en los desenlaces estudiados, con unas OR para mortalidad de 4.26 (IC95\%: 2.10-8.63; $p=0.001$ ), para función retardada del injerto de 8.62 (IC95\%: 4.08-18.21; $p=0.001$ ) y para supervivencia de 2.67 (IC95\%: 1.66-4.30; $p=0.001$ ), con una tendencia a riesgo sin significancia estadística para pérdida del injerto renal y disfunción aguda en el análisis tanto bivariado como multivariado. Hay algunos estudios de supervivencia y mortalidad con resultados similares ${ }^{15,19}$, sin embargo, dichos estudios expresan sus datos finales en frecuencias y porcentaje, con poblaciones menores que la estudiada por nosotros, con 164 y 184 pacientes comparado con 1,118 trasplantados del nuestro ${ }^{19,29}$. Nuestro estudio no analizó otros factores relacionados fuertemente con el donante fallecido, como son la isquemia fría o contar con criterios extendidos o ampliados para muerte; en este caso, hay estudios que hablan claramente de la relación entre un mayor tiempo de isquemia fría y la función retardada del injerto, y de los criterios extendidos para muerte en el caso del donante fallecido ${ }^{30}$.

La edad del receptor del trasplante renal también se ha estudiado. En nuestro estudio, ser mayor de 50 años al momento del trasplante renal fue estadísticamente un riesgo de mortalidad, con OR de 4.16 (IC95\%: 2.07-8.37; $p=0.001$ ), así como de función retardada y supervivencia; además de presentar una tendencia a riesgo de pérdida del injerto renal, sin ser significativo el resultado. La edad se ha estudiado mucho en el receptor y el donante, con riesgo incrementado si este tiene más de 65 años: hazard ratio (HR): 2.59, IC95\%: 1.12-6.00) y HR: 5.65, IC95\%: 2.31-13.79, respectivamente ${ }^{31}$. Se ha demostrado también riesgo con cohortes de edad distintas.

El sexo femenino demostró estadísticamente ser protector para disfunción aguda retardada en el injerto renal (OR: 0.57; IC95\%: 0.33-0.97), sin presentar significancia estadística para los otros cinco rubros. El sexo representa un papel en la incidencia y la progresión de afecciones y del comportamiento del trasplante renal, sobre todo al modificar la farmacocinética y la farmacodinamia de los inmunosupresores; sin embargo, existen muchas investigaciones que permitan determinar el verdadero impacto en este aspecto ${ }^{32}$. Carrero et al..$^{33}$ establecen que el sexo femenino es afectado mayormente en la enfermedad renal crónica terminal en comparación con los hombres, pero no es definido claramente el involucro del sexo en el contexto del trasplante renal.

En el caso del factor de sobrepeso hay controversia, debido a que algunos estudios lo mencionan como un riesgo y otros han encontrado que no lo es. No hay un consenso sobre los efectos de la obesidad 
en los pacientes antes y después del trasplante renal, y estudios relacionados con la obesidad y que han tenido diferentes puntos de vista sobre el tema revelan la necesidad de más investigación ${ }^{34}$. En nuestro estudio, el sobrepeso pretrasplante renal mostró una significancia estadística en los desenlaces clínicos únicamente para la función retardada del injerto, con una OR de 2.4 (IC95\%: 1.30-4.45; $p=0.005$ ). Hay estudios que afirman, en el tema de la obesidad, mejorar el enfoque para el manejo adecuado de candidatos y receptores con algún grado de exceso de peso $^{35}$.

Por último, los dos únicos factores que no presentaron una significancia estadística fueron la dicotomización por FEVI en menos y más del $50 \%$, así como la disfunción diastólica detectada dentro de protocolo de trasplante renal; no obstante, con una tendencia de riesgo en ambos para pérdida del injerto renal y para supervivencia. Esto se explica en parte por la complejidad del sistema cardiovascular, ya que hay efectos de protección proaterogénicos que están compensados con un efecto deletéreo de los fármacos inmunosupresores al endotelio, y hoy toma además relevancia el efecto inmunológico con la inclusión de anticuerpos donante-específicos y su daño directo endotelial en el aloinjerto y sistémico, lo que lleva a la búsqueda de marcadores que orienten a una mejor individualización de los inmunosupresores que no modifiquen la condición cardiaca y endotelial ${ }^{36}$.

\section{Conclusiones}

Se trata de uno de los mayores estudios retrospectivos en México, con 1118 trasplantes renales estudiados y vigilados a lo largo de 12 meses en cuanto a cinco desenlaces: mortalidad, pérdida del injerto renal, función retardada del injerto, disfunción aguda y supervivencia. Se ha encontrado significancia de tres factores de riesgo para dichos desenlaces: recibir el trasplante de un donante fallecido, edad del receptor $>50$ años y uso de agente policlonal en la inducción, que está asociado más a riesgo inmunológico alto. La supervivencia a los 12 meses fue del 90.25\% (1009 pacientes vivos con injerto funcional), la mortalidad del $4.65 \%$ (52 pacientes), la pérdida del injerto del $5.09 \%$ (57 pacientes regresaron a diálisis peritoneal o hemodiálisis), la función retardada del injerto del $5.72 \%$ (64 pacientes) y hubo 103 pacientes $(9.21 \%$ ) con disfunción aguda del injerto y recuperación a lo largo de los 12 meses de seguimiento; por último, 122 pacientes (10.91\%) presentaron rechazo.
En relación con la supervivencia del injerto, se observó que la pérdida de este tuvo una marcada evolución por pacientes que presentaron fallas en la funcionalidad inicial, disfunción aguda o rechazo; en cambio, en la supervivencia del paciente (mortalidad) se observó una relación directa con la sepsis, sobre todo por el gran número de pacientes con neumonía (30 casos de los 52 que fallecieron, $57.69 \%$ ), y también hubo problemas infecciosos abdominales y cardiovasculares.

El sexo femenino se encontró como protección para disfunción aguda del injerto, sin tener un impacto en los otros cuatro desenlaces. El sobrepeso influyó como factor de riesgo en la función retardada del injerto. Los factores $\mathrm{FEVI}<50 \%$ y disfunción diastólica no impactaron estadísticamente en ningún desenlace; sin embargo, sí se observó una tendencia a ser de riesgo para pérdida del injerto y disfunción aguda.

\section{Agradecimientos}

Los autores agradecen a las unidades de trasplante renal por las facilidades otorgadas para la realización de este escrito.

\section{Financiamiento}

Los autores declaran que no se recurrió a ningún financiamiento para la realización de este estudio.

\section{Conflicto de intereses}

Todos los autores declaran no tener ningún conflicto de intereses.

\section{Responsabilidades éticas}

Protección de personas y animales. Los autores declaran que para esta investigación no se han realizado experimentos en seres humanos ni en animales.

Confidencialidad de los datos. Los autores declaran que han seguido los protocolos de su centro de trabajo sobre la publicación de datos de pacientes.

Derecho a la privacidad y consentimiento informado. Los autores declaran que en este artículo no aparecen datos de pacientes.

\section{Bibliografía}

1. Treviño-Becerra A. Insuficiencia renal crónica: enfermedad emergente, catastrófica y por ello prioritaria. Cir Cir. 2004;72:3-4.

2. Arrieta J. Evaluación económica del tratamiento sustitutivo renal (hemodiálisis, diálisis peritoneal y trasplante) en España. Nefrologia. 2010;1(Supl Ext 1):37-47. 
3. Loza-Concha R, Quispe A. Costo-utilidad del trasplante renal frente a la hemodiálisis en el tratamiento de la insuficiencia renal crónica terminal en un hospital peruano. Rev Peru Med Exp Salud Publica. 2011;28:432-9.

4. Acosta P, Chaparro L, Rey C. Calidad de vida y estrategias de afrontamiento en pacientes con insuficiencia renal crónica sometidos a hemodiálisis, diálisis peritoneal 0 trasplante renal. Rev Colom Psico. 2008;17:9-26.

5. García H, Calvanese N. Calidad de vida percibida y locus de control en pacientes con tratamiento sustitutivo de la función renal: diálisis y trasplante renal. Anales de la Universidad Metropolitana. 2007;7:205-22.

6. Khalkhali HR, Ghafari A, Hajizadeh E, Kazemnejad A. Risk factors of long-term graft loss in renal transplant recipients with chronic allograft dysfunction. Exp Clin Transplant. 2010;4:277-82.

7. Dib-Kuri A, Aburto-Morales S, Espinosa-Álvarez A, Sánchez-Ramírez O. Trasplante de órganos y tejidos en México. Rev Invest Clin. 2005:57:163-9.

8. Moreso F, Hernández D. ¿Ha mejorado la supervivencia del injerto tras el trasplante renal en la era de la moderna inmunosupresion? Nefrologia. 2013;33:14-26.

9. Schold JD, Kaplan B, Baliga RS, Meier-Kriesche HU. The broad spectrum of quality in deceased donor's kidneys. Am J Transplant. 2005;(4 pt 1):757-65.

10. Rao PS, Schaubel DE, Guidinger MK, Andreoni KA, Wolfe RA, Merion RM, et al. A comprehensive risk quantification score for deceased donor kidneys: The Kidney Donor Risk Index. Transplantation. 2009:88:231-6

11. Irish WD, McCollum DA, Tesi RJ, Owen AB, Brennan DC, Bailly JE, et al. Nomogram for predicting the likelihood of delayed graft function in cadaveric renal transplant recipients independent of acute rejection. J Am Soc Nephrol. 2003;14:2967-74.

12. Amato D, Álvarez-Aguilar C, Castañeda-Limones R, Rodríguez E, Ávila-Díaz M, Arreola F, et al. Prevalence of chronic kidney disease in an urban Mexican population. Kidney Int. 2005;68(Suppl 97):S11-7.

13. Cueto-Manzano A, Cortés-Sanabria L, Martínez-Ramírez $\mathrm{H}$, Rojas-Campos E, Gómez-Navarro B, Castillero-Manzano M. Prevalence of chronic kidney disease in an adult population. Arch Med Res. 2014:45:507-13.

14. Hernández-Rivera JCH, Damián-Mojica $\mathrm{O}$, Salazar-Mendoza M, Sánchez-Barbosa L, Serrano-Alejandri L, Silva-Rueda RI. Factors that influence the attitude of the population to be a donor in Mexico. Transplant Proc. 2020;52:1036e1041.

15. Crespo M, Esforzado N, Ricart M, Oppenheimer F. Resultados a largo plazo del trasplante renal de donante vivo: supervivencia de injerto y receptor. Arch Esp Urol. 2005;58:537-42.

16. Espinoza-Pérez R, Rodríguez-Gómez R, Cruz-López M, Silva-Rueda R, Bautista-Olayo R, Reyes-Díaz E, et al. Uncommon complications in the first year after renal transplantation. Transplant Proc. 2020;52:1163e1168.

17. Cruz-Santiago J, Velázquez-Zambrano $\mathrm{C}$, Escamilla-Galindo $\mathrm{P}$, Díaz-Rosas G, Rojas-Rodríguez F, Venegas-Vera A, et al. Change in the pattern of post-transplantation anemia in kidney receptors: sex role in recipients and type of donor. Transplant Proc. 2020; 52:1163e1168

18. Rojas-Rodríguez F, Cruz-Santiago J, Meza-Jiménez G, Carreño-Rodríguez Y, Robledo-Meléndez A, Medina-Uicab $C$, et al. Post-transplant cholesterol and triglyceride behavior: effects of sex, age of the recipient, and type of donor. Transplant Proc. 2020;52:1157e1162.
19. Hernández-Rivera J, Salazar-Mendoza M, Pérez-López M, González-Ramos J, Espinoza-Pérez R, Martínez-Álvarez J, et al. Alelos HLA más comunes y asociados con riesgo o protección en enfermedad renal crónica de etiología no determinada. Gac Med Mex. 2019;155:243-8.

20. Hernández D, Alonso-Titos J, Armas-Padrón A, Ruiz-Esteban $P$, Cabello M, López V, et al. Mortality in elderly waiting-list patients versus age-matched kidney transplant recipients: ¿where is the risk? Kidney Blood Press Res. 2018:43:256-75.

21. Schold J, Srinivas TR, Sehgal AR, Meier-Kriesche HU. Half of kidney transplant candidates who are older than 60 years now placed on the waiting list will die before receiving a deceased-donor transplant. Clin $\mathrm{J}$ Am Soc Nephrol. 2009:4:1239-45.

22. Jiménez L, Franco A, Rivera F, Olivares J. Evolución de la función renal en el trasplante renal. Factores predictivos del deterioro funcional. Nefrología. 2001;21:295-304.

23. Vanichanan J, Udomkarnjananun $\mathrm{S}$, Avihingsanon $\mathrm{Y}$, Jutivorakool $\mathrm{K}$. Common viral infections in kidney transplant recipients. Kidney Res Clin Pract. 2018;37:323-37.

24. Masutani K. Viral infections directly involved in kidney allograft function. Nephrology. 2018;23(Suppl 2):31-7.

25. Pérez-Gutiérrez A, Morales-Buenrostro L, Vilatobá-Chapa M, Mendoza-De la Garza A, Vega-Vega O, Gabilondo-Pliego B, et al. Factores de riesgo para el desarrollo de función retardada del injerto en receptores de trasplante renal de donante fallecido y su impacto en la supervivencia de paciente e injerto. Rev Invest Clin. 2013;65:109-15.

26. Otaibi T, Ahmadpoor P, Dyab A, Habhab W, Reza M, Nafar M, et al. Delayed graft function in living-donor kidney transplant: a Middle Eastern perspective. Exp Clin Transplant. 2016;1:1-11.

27. Gill J, Dong J, Rose C, Gill J. The risk of allograft failure and the survival benefit of kidney transplantation are complicated by delayed graft function. Kidney Int. 2016;89:1331-6.

28. United States Renal Data System (USRDS). Annual Data Report. End Stage Renal Disease. 5: Mortality. Disponible en: https://adr.usrds. org/2020/end-stage-renal-disease/5-mortality

29. Dávila F, Luna R, Pardo D. Análisis de supervivencia del injerto post-trasplante renal. Rev Colomb Nefrol. 2015;2:96-101.

30. Fragoso S, Espinoza R, Cruz M, Moreno L, Hernández J. Impact of cold ischemia time in clinical outcomes in deceased donor renal transplant. Transplant Proc. 2020;52:1118e1122.

31. Veroux M, Grosso G, Corona D, Mistretta A, Giaquinta A, Giuffida G, et al. Age is an important predictor of kidney transplantation outcome. Nephrol Dial Transplant. 2012;27:1663-71.

32. Momper J, Misel M, McKay D. Sex differences in transplantation. Transplant Rev. 2017;31:145-50.

33. Carrero J, Hecking M, Chesnaye N, Jager K. Sex and gender disparities in the epidemiology and outcomes of chronic kidney disease. Nature Rev Nephrol. 2018:14:151-64.

34. Ates D, Cebeci F. Obesity in the kidney transplant process. Exp Clin Transplant. 2018;16(Suppl 1):61-3.

35. Glicklich D, Mustafa M. Obesity in kidney transplantation: impact on transplant candidates, recipients and donors. Cardiol Rev. 2019;27:63-72.

36. Laecke S, Malfait T, Schepers E, Van Biesen W. Cardiovascular disease after transplantation: an emerging role of the immune system. Transpl Int. 2018;31:689-99. 